\title{
Investigating student understanding of the stationary state wavefunction for a system of identical particles
}

\author{
Christof Keebaugh \\ Department of Physics and Astronomy, Franklin and Marshall College, Lancaster, PA 17603 \\ Emily Marshman \\ Department of Physics and Astronomy, Community College of Allegheny County, Pittsburgh, PA 15260 \\ Chandralekha Singh \\ Department of Physics and Astronomy, University of Pittsburgh, Pittsburgh, PA 15260
}

We discuss an investigation of student difficulties with concepts related to the many-particle stationary state wavefunction for a system of non-interacting fermions or bosons in cases in which the many-particle stationary state wavefunction can be written as the product of the spatial and spin parts. The investigation was carried out in advanced quantum mechanics courses by administering free-response and multiple-choice questions and conducting individual interviews with students. We find that students share many common difficulties related to these concepts. Many students struggled to write a many-particle stationary state wavefunction consistent with the symmetrization requirements for the system, i.e., a completely antisymmetric wavefunction for a system of fermions or a completely symmetric wavefunction for a system of bosons. We discuss the common difficulties pertaining to these concepts that can be used as a guide to develop research-validated learning tools.

\footnotetext{
2020 PERC Proceedings edited by Wolf, Bennett, and Frank; Peer-reviewed, doi.org/10.1119/perc.2020.pr.Keebaugh Published by the American Association of Physics Teachers under a Creative Commons Attribution 4.0 license. Further distribution must maintain the cover page and attribution to the article's authors.
} 


\section{INTRODUCTION AND BACKGROUND}

Quantum mechanics (QM) is a particularly challenging subject for upper-level undergraduate and graduate students in physics as evidenced by many investigations, e.g., see Refs. [1-20]. We have been involved in a number of research studies aimed at investigating student reasoning in QM and improving student understanding of QM [21-35]. However, there have been few investigations into student difficulties with fundamental concepts involving a system of identical particles. Through researching students' understanding and reasoning about a system of identical particles, we have found many common student difficulties that can hinder the development of a consistent and coherent knowledge structure pertaining to these concepts.

In nature, there are two general types of particles: fermions with a half-integer spin quantum number (e.g., electrons and protons) and bosons with an integer spin quantum number (e.g., photons and mesons). A system of $N$ identical particles consists of $N$ particles of the same type (e.g., electrons). For a system of identical particles in classical mechanics (e.g., five identical tennis balls), each particle can be distinguished from all the other particles. In contrast, in quantum mechanics, identical particles are indistinguishable and there is no measurement that can be performed to distinguish these identical particles from one another. For example, if the coordinates of two identical particles are interchanged, there is no physical observable that would reflect this interchange. Furthermore, one property that distinguishes these two types of particles is that two or more bosons can occupy the same single-particle quantum state, but two or more fermions can never occupy the same single-particle quantum state. The restriction for fermions is known as the Pauli exclusion principle and is consistent with a system of fermions having a completely antisymmetric wavefunction. To reflect the indistinguishability of these identical particles and make the statistical properties of fermions and bosons consistent with observations, the wavefunction for a system of identical fermions must be completely antisymmetric and the wavefunction for a system of identical bosons must be completely symmetric. Here we focus on student difficulties with the many-particle stationary state wavefunction that is a solution to the Time-Independent Schrödinger Equation (TISE) for a system of non-interacting identical particles. Unless otherwise stated, throughout, we refer to the stationary state wavefunction as the wavefunction.

Even though the spatial and spin parts of the wavefunction can be entangled in many situations, we will only consider many-particle wavefunctions $\Psi\left(x_{1}, x_{2}, x_{3}, \ldots, m_{s_{1}}, m_{s_{2}}, m_{s_{3}}, \ldots\right)=$ $\psi\left(x_{1}, x_{2}, x_{3}, \ldots\right) \chi\left(m_{s_{1}}, m_{s_{2}}, m_{s_{3}}, \ldots\right) \quad$ in one spatial dimension that can be written as the product of the spatial part of the wavefunction $\psi\left(x_{1}, x_{2}, x_{3}, \ldots\right)$ and the spin part of the wavefunction $\chi\left(m_{s_{1}}, m_{s_{2}}, m_{s_{3}}, \ldots\right)$, in which $x_{i}$ denotes the spatial coordinate and $m_{s_{i}}$ denotes the $z$-component of spin quantum number of the $i^{\text {th }}$ particle.

The spatial part of the wavefunction of a system of two non-interacting identical particles has terms such as $\psi_{n_{a}}\left(x_{i}\right) \psi_{n_{b}}\left(x_{j}\right)$, where $\psi_{n_{a}}\left(x_{i}\right)$ and $\psi_{n_{b}}\left(x_{j}\right)$ are the singleparticle wavefunction for the $i^{t h}$ particle with coordinate $x_{i}$ in the state $n_{a}$ and for the $j^{\text {th }}$ particle with coordinate $x_{j}$ in the state $n_{b}$, respectively.

If we have a system of two non-interacting identical fermions, the two-particle stationary state wavefunction must be completely antisymmetric. There are two ways to construct a completely antisymmetric wavefunction: the spatial part of the wavefunction could be completely symmetric and the spin part of the wavefunction could be completely antisymmetric or the spatial part of the wavefunction could be completely antisymmetric and the spin part of the wavefunction could be completely symmetric. If we have a system of two non-interacting identical bosons, the two-particle stationary state wavefunction must be completely symmetric. There are two ways to construct a completely symmetric wavefunction: the spatial and spin parts of the wavefunction could both be completely symmetric or the spatial and spin parts of the wavefunction could both be completely antisymmetric.

When considering the spin part of the wavefunction for a single-particle, we use the notation $\left|s_{i}, m_{s_{i}}\right\rangle$ (in which $s_{i}$ and $m_{s_{i}}$ are the quantum numbers corresponding to the total spin and z-component of the spin for the $i^{t h}$ particle, respectively). The states $\left|s_{1}, m_{s_{1}}\right\rangle$ are eigenstates of $\hat{S}_{1}^{2}$ and $\hat{S}_{1 z}$ and the states $\left|s_{2}, m_{s_{2}}\right\rangle$ are eigenstates of $\hat{S}_{2}^{2}$ and $\hat{S}_{2 z}$. We use the following abbreviated notation for a spin- $1 / 2$ particle, e.g., an electron: $|\uparrow\rangle_{1}=\left|s_{1}, m_{s_{1}}\right\rangle=|1 / 2,1 / 2\rangle_{1}$ and $|\downarrow\rangle_{1}=$ $\left|s_{1}, m_{s_{1}}\right\rangle=|1 / 2,-1 / 2\rangle_{1}$ for electron 1 in the "spin up" and "spin down" state, respectively, and $|\uparrow\rangle_{2}=\left|s_{2}, m_{s_{2}}\right\rangle=$ $|1 / 2,1 / 2\rangle_{2}$, and $|\downarrow\rangle_{2}=\left|s_{2}, m_{s_{2}}\right\rangle=|1 / 2,-1 / 2\rangle_{2}$ for electron 2 in the "spin up" and "spin down" state, respectively.

When considering the spin part of the wavefunction for the two spin-1/2 particles in the uncoupled representation in the product space, we will use the notation $|\uparrow \uparrow\rangle=|\uparrow\rangle_{1}|\uparrow\rangle_{2}$, $|\uparrow \downarrow\rangle=|\uparrow\rangle_{1}|\downarrow\rangle_{2},|\downarrow \uparrow\rangle=|\downarrow\rangle_{1}|\uparrow\rangle_{2}$, and $|\downarrow \downarrow\rangle=|\downarrow\rangle_{1}|\downarrow\rangle_{2}$ for the basis states. In order to satisfy the symmetrization requirement for a system of two spin-1/2 particles, the spin part of the wavefunction must be either completely symmetric or antisymmetric so that the overall wavefunction is antisymmetric. In the case of two spin-1/2 particles, $|\uparrow \uparrow\rangle,|\downarrow \downarrow\rangle, \frac{1}{\sqrt{2}}(|\uparrow \downarrow\rangle+|\downarrow \uparrow\rangle)$ are completely symmetric spin states of the two-fermion wavefunction and often referred to as the "triplet" states. The state $\frac{1}{\sqrt{2}}(|\uparrow \downarrow\rangle-|\downarrow \uparrow\rangle)$ is the completely antisymmetric normalized spin state of the twofermion wavefunction, often referred to as the "singlet" state.

The following are examples of completely antisymmetric normalized many-particle stationary state wavefunctions for a system of two spin-1/2 fermions in which the spatial part of the wavefunction is antisymmetric and the spin part of the wavefunction is symmetric (or vice-versa, assume $\left.n_{1} \neq n_{2}\right): \Psi\left(x_{1}, x_{2}, m_{s_{1}}, m_{s_{2}}\right)=\frac{1}{\sqrt{2}}\left\{\psi_{n_{1}}\left(x_{1}\right) \psi_{n_{2}}\left(x_{2}\right)-\right.$ $\left.\psi_{n_{2}}\left(x_{1}\right) \psi_{n_{1}}\left(x_{2}\right)\right\}|\uparrow\rangle_{1}|\uparrow\rangle_{2}$ and $\Psi\left(x_{1}, x_{2}, m_{s_{1}}, m_{s_{2}}\right)=$ $\psi_{n_{1}}\left(x_{1}\right) \psi_{n_{1}}\left(x_{2}\right) \frac{1}{\sqrt{2}}\left\{|\uparrow\rangle_{1}|\downarrow\rangle_{2}-|\downarrow\rangle_{1}\left|\uparrow_{2}\right\rangle\right\}$. 
For a spin-1 boson, $\left|s_{i}, m_{s_{i}}\right\rangle=\{|1,-1\rangle,|1,0\rangle,|1,1\rangle\}$ for each particle. When considering the spin part of the wavefunction for two spin-1 particles in the uncoupled representation in the product space $(3 \times 3=9$ dimensional), we will use the notation $|1,1\rangle_{1}|1,1\rangle_{2}$, $|1,1\rangle_{1}|1,0\rangle_{2},|1,1\rangle_{1}|1,-1\rangle_{2},|1,0\rangle_{1}|1,1\rangle_{2},|1,0\rangle_{1}|1,0\rangle_{2}$, $|1,0\rangle_{1}|1, \quad-1\rangle_{2}, \quad|1,-1\rangle_{1}|1, \quad 1\rangle_{2}, \quad|1,-1\rangle_{1}|1, \quad 0\rangle_{2}$, and $|1,-1\rangle_{1}|1,-1\rangle_{2}$ for the basis states.

The wavefunction for a system of identical bosons must be completely symmetric. The spatial and spin parts of the many-particle stationary state wavefunction can either be both symmetric or both antisymmetric. For example, the following are examples of completely symmetric many-particle stationary state wavefunctions for a system of two spin-1 bosons: $\Psi\left(x_{1}, x_{2}, m_{s_{1}}, m_{s_{2}}\right)=\psi_{n_{1}}\left(x_{1}\right) \psi_{n_{1}}\left(x_{2}\right)|1, \quad 1\rangle_{1}|1, \quad 1\rangle_{2}$ and $\Psi\left(x_{1}, x_{2}, m_{s_{1}}, m_{s_{2}}\right)=\frac{1}{\sqrt{2}}\left\{\psi_{n_{1}}\left(x_{1}\right) \psi_{n_{2}}\left(x_{2}\right)-\right.$ $\left.\psi_{n_{2}}\left(x_{1}\right) \psi_{n_{1}}\left(x_{2}\right)\right\} \frac{1}{\sqrt{2}}\left\{|1,1\rangle_{1}|1,0\rangle_{2}-|1,0\rangle_{1}|1,1\rangle_{2}\right\}$ in which both the spatial and spin parts of the wavefunction are symmetric or antisymmetric (assume $n_{1} \neq n_{2}$ ), respectively.

\section{METHODOLOGY}

Student difficulties with determining the many-particle stationary state wavefunction for a system of identical fermions or bosons were first investigated using three years of data involving responses to open-ended and multiplechoice questions administered after traditional instruction in relevant concepts from 57 upper-level undergraduate students in a junior/senior level QM course and 30 graduate students in the second semester of the graduate core QM course. Additional insight concerning these difficulties was gained from responses of 14 students during a total of 81 hours of individual "think-aloud" interviews.

We discuss student responses to several questions that were posed either as in class clicker questions or as open-ended quiz questions. Additional insight into these difficulties was gleaned during the individual think-aloud interviews in which students were asked questions pertaining to these issues. To probe whether students are able to identify and generate a many-particle stationary state wavefunction including spin, the following two questions were posed to the students. Questions Q1 and Q2 were posed on a quiz following traditional instruction on concepts involving a system of identical particles to 30 graduate students and 25 undergraduate students. Students were told that the particles are confined in one spatial dimension and that $\psi_{n_{1}}, \psi_{n_{2}}$, etc., are the single-particle stationary state wavefunctions. Below, we discuss the questions students were asked: Q1. Write one possible spatial part of the wavefunction for two indistinguishable spin-1 bosons if the spin part of the wavefunction (expressed in terms of the uncoupled

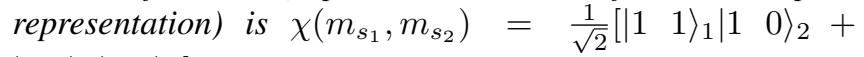
$\left.\left|\begin{array}{lll}1 & 0 & \rangle_{1}\end{array}\right| \begin{array}{ll}1 & 1\end{array}\right\rangle_{2}$ ]. If it is not possible to write a spatial part of the wavefunction with the given spin part of the wavefunction, write "not possible" and state the reason.

The overall wavefunction for the two indistinguishable bosons must be completely symmetric. Since the spin part of the wavefunction given in Q1 is symmetric, the spatial part of the wavefunction must also be symmetric to ensure that the overall wavefunction is completely symmetric. Two possible symmetric spatial states for the two spin-1 bosons are $\psi\left(x_{1}, x_{2}\right)=\psi_{n_{1}}\left(x_{1}\right) \psi_{n_{1}}\left(x_{2}\right)$ and (assume $\left.n_{1} \neq n_{2}\right)$ $\psi\left(x_{1}, x_{2}\right)=\frac{1}{\sqrt{2}}\left[\psi_{n_{1}}\left(x_{1}\right) \psi_{n_{2}}\left(x_{2}\right)+\psi_{n_{2}}\left(x_{1}\right) \psi_{n_{1}}\left(x_{2}\right)\right]$.

Q2. Write one possible spin part of the wavefunction for two electrons if the spatial part of the wavefunction is $\psi\left(x_{1}, x_{2}\right)=\frac{1}{\sqrt{2}}\left[\psi_{n_{1}}\left(x_{1}\right) \psi_{n_{2}}\left(x_{2}\right)+\psi_{n_{2}}\left(x_{1}\right) \psi_{n_{1}}\left(x_{2}\right)\right]$. If it is not possible to write a spin part of the wavefunction with the given spatial part of the wavefunction, write "not possible" and state the reason.

The overall wavefunction for the two electrons must be completely antisymmetric. Since the spatial part of the wavefunction given in Q2 is symmetric, the spin part of the wavefunction must be antisymmetric to ensure that the overall wavefunction is completely antisymmetric. The antisymmetric spin state for the two spin-1/2 fermions is $\chi\left(m_{s_{1}}, m_{s_{2}}\right)=\frac{1}{\sqrt{2}}[|\uparrow \downarrow\rangle-|\downarrow \uparrow\rangle]$.

Q3 below was posed as an in-class clicker question to 16 undergraduate students in a junior/senior level undergraduate quantum mechanics course following instruction on identical particles. The students first answered the question individually and then answered it a second time after discussing the question with their peers in small groups.

Q3. Choose all of the following statements that are correct about bosons. (1) The spin of a boson is an integer. (2) The overall wavefunction of identical bosons can be antisymmetric. (3) Two bosons cannot occupy the same singleparticle state.

Only option (1) is correct for question Q3. Option (2) is incorrect because the overall wavefunction for a system of identical bosons MUST be symmetric and option (3) is incorrect because two or more bosons can occupy the same single-particle state.

Question Q4 was posed during the think-aloud interviews to investigate students' proficiency at identifying whether the spin part of a wavefunction is a symmetric or antisymmetric wavefunction. The question focuses on a system of two spin-1/2 particles $\left(s_{1}=1 / 2, s_{2}=1 / 2\right)$. The students were familiar with the shorthand notation $|\uparrow \uparrow\rangle=|\uparrow\rangle_{1}|\uparrow\rangle_{2}$, $|\uparrow \downarrow\rangle=|\uparrow\rangle_{1}|\downarrow\rangle_{2},|\downarrow \uparrow\rangle=|\downarrow\rangle_{1}|\uparrow\rangle_{2}$, and $|\downarrow \downarrow\rangle=|\downarrow\rangle_{1}|\downarrow\rangle_{2}$.

Q4. For the spin part of the wavefunction (spin state) of a two-particle system given below, identify whether the spin state is symmetric, antisymmetric, or neither symmetric nor antisymmetric with respect to exchange of the two particles. Explain your reasoning. (a) $|\uparrow \uparrow\rangle$ (b) $|\downarrow \downarrow\rangle$ (c) $|\uparrow \downarrow\rangle$ (d) $\frac{1}{\sqrt{2}}(|\uparrow \downarrow\rangle+|\downarrow \uparrow\rangle)(\mathrm{e}) \frac{1}{\sqrt{2}}(|\uparrow \downarrow\rangle-|\downarrow \uparrow\rangle)$

In Q4, options (a), (b), and (d) are symmetric spin states (triplet states) since exchanging the two particles results in the same state. Option (e) in Q4 is an antisymmetric spin state (singlet state) since exchanging the two particles results in the original state multiplied by -1 . Option (c) in Q4 is neither a symmetric nor antisymmetric spin state. 
TABLE I. The percentages of graduate $(N=30)$ and undergraduate $(N=25)$ students who correctly answered questions Q1 and Q2 after traditional instruction.

\begin{tabular}{|c|c|c|c|}
\hline Question & Type of Particle & Graduate (\%) & Undergraduate (\%) \\
\hline Q1 & Bosons & 33 & 48 \\
\hline Q2 & Electrons & 30 & 44 \\
\hline
\end{tabular}

\section{STUDENT DIFFICULTIES}

Many students struggled to recognize and generate the completely symmetric many-particle wavefunction for a system of indistinguishable bosons in question Q1 and the completely antisymmetric many-particle wavefunction for a system of indistinguishable fermions in question Q2. Table I summarizes the percentages of students who answered questions Q1 and Q2 correctly for a system of two indistinguishable fermions or bosons after traditional instruction.

Below, we discuss several of difficulties students had that interfered with their ability to write the completely symmetric/antisymmetric many-particle stationary state wavefunction for a system of indistinguishable particles.

A. Difficulty applying Pauli's exclusion principle correctly for a system of identical fermions: Some students struggled to realize that Pauli's exclusion principle states that no two fermions can be in the same single-particle state. Students with this type of difficulty often incorrectly overgeneralized the Pauli exclusion principle to state that no two fermions can occupy the same spatial state or the same spin state. Often students had difficulty realizing that two fermions can be in the same spatial state if they are in different spin states or vice-versa, so that the overall wavefunction is antisymmetric. During the interview, several students incorrectly applied the Pauli exclusion principle when considering a separable many-particle stationary state wavefunction that can be expressed as the direct product of the spatial and the spin parts of the wavefunction. For example, one interviewed student correctly stated that no two fermions can be in the same single-particle state, but then went on to incorrectly claim that "this means two fermions could not exist in the same single-particle stationary state (pointing to a case in which they were in the same spatial state)". This student and others with this type of difficulty often had difficulty realizing that if the two spin$1 / 2$ fermions are in different spin states then it is possible for the two fermions to be in the same single-particle spatial state, i.e. $\psi_{n_{1}}\left(x_{1}\right) \psi_{n_{1}}\left(x_{2}\right)$ is a possible spatial part of the wavefunction.

By a similar argument, several students incorrectly claimed that two fermions could not exist in the same spin state as this too would violate the Pauli exclusion principle. For example, some interviewed students claimed that $|\uparrow \uparrow\rangle$ and $|\downarrow \downarrow\rangle$ were not possible spin states for the system of two spin-1/2 fermions regardless of whether the two fermions were in different single-particle spatial states. These students did not consider the fact that the two fermions could be in a two-particle state consisting of product states of spatial states involving anti-symmetric spatial wavefunction and symmetric spin states.

B. Difficulty with the symmetrization requirements for the overall many-particle stationary state wavefunction: Nature demands that the many-particle wavefunction for a system of indistinguishable bosons be completely symmetric and the many-particle wavefunction for a system of indistinguishable fermions be completely antisymmetric. Therefore, in order to identify and generate a manyparticle wavefunction for a system of indistinguishable particles, students must be able to determine a completely symmetric/antisymmetric wavefunction involving both spatial and spin degrees of freedom. However, some students struggled to correctly identify the appropriate symmetrization requirement for a system of identical particles. Other students had difficulty determining whether the spatial part of the wavefunction or the spin part of the wavefunction is symmetric or antisymmetric when considering each part of the wavefunction separately. In interviews, students with this type of difficulty often were not able to correctly identify the symmetry of the overall wavefunction that included both the spatial and spin parts of the wavefunction. Lastly, some students struggled to construct the overall many-particle stationary state wavefunction due to the fact that it is the product of the spatial and spin parts.

C. Difficulty identifying the correct symmetrization requirement for a system of identical particles: Students also had difficulty identifying that the many-particle wavefunction for a system of identical bosons must be completely symmetric. For example, in response to Q3, $43 \%$ of the undergraduate students incorrectly answered that the overall wavefunction of identical bosons can be antisymmetric (option (2)). Even after peer discussion, 31\% again incorrectly selected option (2) as correct. Written explanations and interviews suggest that these students knew that the many-particle wavefunction for a system of identical particles (bosons or fermions) must obey a symmetrization requirement, but could not correctly identify which symmetrization requirement corresponds to which particle. For example, one interviewed student in response to questions Q1 and Q2 stated that "there is a symmetrization requirement for fermions and a different symmetrization requirement for bosons." But the student was unable to recognize the appropriate symmetrization requirements and wrote the same symmetric wavefunction for both a system of indistinguishable fermions and indistinguishable bosons. In response to $\mathrm{Q} 1$, some students attempted to generate a completely antisymmetric wavefunction for a system of identical bosons. Similarly, in response to Q2, some students generated a completely symmetric wavefunction for a system of identical fermions. Additionally, $67 \%$ of the graduates and $28 \%$ of undergraduate students incorrectly provided a symmetric spin part of the wavefunction in response to Q2 resulting in an overall symmetric many-particle wavefunction for the two electrons. 
Identifying the correct symmetrization requirement for the overall many-particle stationary state wavefunction is challenging due in part to the fact that one must consider the symmetry of both the spatial and spin parts of the wavefunction before determining the overall symmetry of the many-particle stationary state wavefunction. This can be confusing for students who simply memorized the appropriate combinations of the spatial and spin parts of the wavefunction rather than developing an understanding of the symmetry of a wavefunction comprised of two separate parts.

D. Incorrectly determining the symmetry based on the appearance of a $+/-$ sign in the many-particle wavefunction: Some students incorrectly applied a heuristic by which they claimed that a wavefunction is symmetric if the wavefunction is written in terms of a sum. These students simply looked for all " + " signs to determine that a wavefunction is symmetric. They claimed that any wavefunction written as terms added together is a symmetric wavefunction. By a similar logic, these same students looked for a "-" sign to determine whether a given wavefunction is antisymmetric. Some claimed that any wavefunction that had at least one negative sign was antisymmetric. For example, in response to question Q4(a), one interviewed student incorrectly claimed that the spin part of the wavefunction given by $|\uparrow \uparrow\rangle$ is neither symmetric nor antisymmetric as "the wavefunction is not a sum so it can't be symmetric and there is not a minus sign, so it can't be antisymmetric." However, the spin part of the wavefunction given by $|\uparrow \uparrow\rangle$ is symmetric as the exchange of the two particles results in the same wavefunction, thus there need not be a plus sign in order for a wavefunction to be symmetric. Other students used similar reasoning when determining the symmetry of the spin part of the wavefunction.

Students with this type of difficulty often struggled to write the overall many-particle stationary state wavefunction for a system of identical particles with the appropriate symmetrization requirement. For example, students who incorrectly claimed that the spin part of the wavefunction $|\uparrow \uparrow\rangle$ is neither symmetric nor antisymmetric often also incorrectly claimed that it is not possible to write a many-particle stationary state wavefunction for two fermions with this spin state.

E. Difficulty identifying that the spatial part is antisymmetric and the spin part is symmetric (or vice versa) for the wavefunction for a system of identical fermions: Some students correctly identified that the many-particle stationary state wavefunction for a system of identical fermions must be completely antisymmetric, but incorrectly claimed that both the spatial and spin parts of the wavefunction must be completely antisymmetric. For example, when asked to determine the spatial part of the wavefunction for two fermions if the spin part of the wavefunction was the singlet state, some students incorrectly claimed that the spatial part of the wavefunction is $\frac{1}{\sqrt{2}}\left[\psi_{n_{1}}\left(x_{1}\right) \psi_{n_{2}}\left(x_{2}\right)-\psi_{n_{2}}\left(x_{1}\right) \psi_{n_{1}}\left(x_{2}\right)\right]$. They struggled to realize that the product of two completely antisymmetric wavefunctions is a completely symmetric wavefunction. During the interviews, some students incorrectly claimed that in order for the overall many-particle stationary state wavefunction to be antisymmetric, both the spatial and spin parts of the wavefunction must be antisymmetric.

F. Difficulty identifying that the spatial and spin parts of the wavefunction can both be antisymmetric for a system of identical bosons: Some students had difficulty realizing that both the spatial and spin parts of the wavefunction can be antisymmetric to produce an overall symmetric wavefunction for a system of identical bosons. A number of students correctly reasoned that the overall many-particle wavefunction for a system of indistinguishable bosons must be completely symmetric. However, some of these students went on to incorrectly claim that both the spatial part and spin part of the wavefunction must be symmetric. These students did not realize that a manyparticle stationary state wavefunction in which both the spatial part and spin part are antisymmetric is completely symmetric.

For example, students were asked to construct the spin part of a two-particle stationary state wavefunction for two spin1 bosons whose spatial part of the wavefunction is given by $\frac{1}{\sqrt{2}}\left[\psi_{n_{1}}\left(x_{1}\right) \psi_{n_{2}}\left(x_{2}\right)-\psi_{n_{2}}\left(x_{1}\right) \psi_{n_{1}}\left(x_{2}\right)\right]$. One interviewed student incorrectly claimed that "it is not possible to write the spin part since the spatial part is antisymmetric. There is no way to make the whole wavefunction symmetric." This student and many others with this type of difficulty did not realize that by choosing an antisymmetric spin part of the wavefunction, the overall two-particle wavefunction would be completely symmetric.

\section{SUMMARY AND FUTURE OUTLOOK}

Our investigation suggests that upper-level undergraduate and graduate students have many common difficulties with fundamental concepts involving a system of non-interacting identical particles. We are using the difficulties found in this context as a guide to develop and validate a Quantum Interactive Learning Tutorial (QuILT). The QuILT focuses on helping students learn that the wavefunction for a system of fermions must be completely antisymmetric and the wavefunction for a system of bosons must be completely symmetric and then construct the many-particle stationary state wavefunction for a system of non-interacting identical particles (fermions or bosons) consistent with the symmetrization requirements. In particular, the QuILT strives to help students understand and operationalize completely symmetric or completely anti-symmetric functions with respect to the exchange of any two particles' coordinates and use this knowledge to construct a many-particle wave function consisting of both spatial and spin parts.

The initial in-class evaluation of the validated QuILT is encouraging.

\section{ACKNOWLEDGEMENTS}

We thank the NSF for award PHY-1806691. 
[1] P. Jolly, D. Zollman, S. Rebello and A. Dimitrova. Visualizing potential energy diagrams, Am. J. Phys. 66, 57 (1998).

[2] C. Singh, Student understanding of quantum mechanics, Am. J. Phys. 69, 885 (2001).

[3] D. Zollman, N. S. Rebello, and K. Hogg, Quantum mechanics for everyone: Hands-on activities integrated with technology, Am. J. Phys. 70, 252 (2002).

[4] R. Muller and H. Wiesner, Teaching quantum mechanics on an introductory level, Am. J. Phys. 70, 200 (2002).

[5] M. Wittmann et al. Investigating student understanding of quantum physics: Spontaneous models of conductivity, Am.J. Phys. 70, 218 (2002).

[6] D. Domert, C. Linder, and A. Ingerman, Probability as a conceptual hurdle to understanding one-dimensional quantum scattering and tunnelling, Eur. J. Phys. 26, 47 (2004).

[7] C. Singh, Student understanding of quantum mechanics at the beginning of graduate instruction, Am. J. Phys. 76, 277 (2008).

[8] S. Y. Lin and C. Singh, Categorization of quantum mechanics problems by professors and students, Euro. J. Phys. 31, 57 (2010).

[9] A. Kohnle et al., Developing and evaluating animations for teaching quantum mechanics concepts, Eur. J. Phys. 31, 1441 (2010).

[10] A. J. Mason and C. Singh, Do advanced students learn from their mistakes without explicit intervention?, Am. J. Phys. 78, 760 (2010).

[11] G. Zhu and C. Singh, Surveying students' understanding of quantum mechanics in one spatial dimension, Am. J. Phys. 80, 252 (2012).

[12] A. Kohnle, I. Bozhinova, D. Browne, M. Everitt, A. Fomins, P. Kok, G. Kulaitis, M. Prokopas, D. Raine and E. Swinbank, A new introductory quantum mechanics curriculum, Eur. J. Phys. 35, 015001 (2014).

[13] E. Gire and E. Price, The structural features of algebraic quantum notations, Phys. Rev. ST PER 11, 020109 (2015).

[14] E. Marshman and C. Singh, Framework for understanding the patterns of student difficulties in quantum mechanics, Phys. Rev. ST PER 11, 020119 (2015).

[15] C. Singh and E. Marshman, Review of student difficulties in upper-level quantum mechanics, Phys. Rev. ST PER 11, 020117 (2015).

[16] G. Passante, P. Emigh, and P. Shaffer, Examining student ideas about energy measurements on quantum states across undergraduate and graduate levels, Phys. Rev. ST PER 11, 020111 (2015).

[17] E. Marshman and C. Singh, Investigating and improving student understanding of the expectation values of observables in quantum mechanics, Eur. J. Phys. 38, 045701 (2017).

[18] V. Dini and D. Hammer, Case study of a successful learner's epistemological framings of quantum mechanics, Phys. Rev. Phys. Educ. Res. 13, 010124 (2017).

[19] E. Marshman and C. Singh, Investigating and improving student understanding of the probability distributions for measuring physical observables in quantum mechanics, Eur. J. Phys. 38, 025705 (2017).
[20] E. Marshman and C. Singh, Investigating and improving student understanding of quantum mechanical observables and their corresponding operators in Dirac notation, Eur. J. Phys. 39, 015707 (2017).

[21] C. Singh, M. Belloni, and W. Christian, Improving students' understanding of quantum mechanics, Physics Today 59, 43 (2006).

[22] C. Singh, Interactive learning tutorials on quantum mechanics, Am. J. Phys. 76, 400 (2008).

[23] G. Zhu and C. Singh, Improving students' understanding of quantum measurement: I. Investigation of difficulties, Phys. Rev. ST PER 8, 010117 (2012).

[24] G. Zhu and C. Singh, Improving students' understanding of quantum mechanics via the Stern-Gerlach experiment, Am. J. Phys. 79, 499 (2011).

[25] G. Zhu and C. Singh, Improving students' understanding of quantum measurement: II. Development of research-based learning tools, Phys. Rev. ST PER 8, 010118 (2012).

[26] G. Zhu and C. Singh, Improving student understanding of addition of angular momentum in quantum mechanics, Phys. Rev. ST PER 9, 010101 (2013).

[27] B. Brown, A. Mason, and C. Singh, Improving performance in quantum mechanics with explicit incentives to correct mistakes, Phys. Rev. PER 12, 010121 (2016).

[28] E. Marshman and C. Singh, Investigating and improving student understanding of quantum mechanics in the context of single photon interference, Phys. Rev. PER 13, 010117 (2017).

[29] R. Sayer, A. Maries and C. Singh, A quantum interactive learning tutorial on the double-slit experiment to improve student understanding of quantum mechanics, Phys Rev PER 13, 010123 (2017).

[30] A. Maries, R. Sayer and C. Singh, Effectiveness of interactive tutorials in promoting "which-path" information reasoning in advanced quantum mechanics, Phys. Rev. PER 13, 020115 (2017).

[31] C. Keebaugh, E. Marshman and C. Singh, Investigating and addressing student difficulties with the corrections to the energies of the hydrogen atom for the strong and weak field Zeeman effect, Eur. J. Phys. 39, 045701 (2018).

[32] C. Keebaugh, E. Marshman and C. Singh, Investigating and addressing student difficulties with a good basis for finding perturbative corrections in the context of degenerate perturbation theory, Eur. J. Phys. 39, 055701 (2018).

[33] C. Keebaugh, E. Marshman and C. Singh, Improving student understanding of corrections to the energy spectrum of the hydrogen atom for the Zeeman effect, Phys. Rev. Phys. Educ. Res. 15, 010113 (2018).

[34] C. Keebaugh, E. Marshman and C. Singh, Improving student understanding of fine structure corrections to the energy spectrum of the hydrogen atom, Am. J. Phys. 87, 594 (2019).

[35] C. Keebaugh, E. Marshman and C. Singh, Improving student understanding of a system of identical particles with a fixed total energy, Am. J. Phys. 87, 583 (2019). 